\title{
Análisis de un modelo de asistencia clínica compartida con internistas en un servicio de cirugía vascular
}

\author{
Analysis of a comanagement clinical model \\ with internists in a vascular surgery department
}

\author{
Rafael Monte Secades, Eva Romay Lema, Iria Iñiguez Vázquez, Ramón Rabuñal Rey, Beatriz Pombo Vide \\ Servicio de Medicina Interna. Hospital Universitario Lucus Augusti. SERGAS. Lugo
}

\section{Resumen}

Objetivo: valorar si la asistencia clínica compartida entre cirujanos vasculares e internistas mejora la calidad de la atención médica prestada a pacientes ingresados en un servicio de cirugía vascular.

Material y método: se analizaron diversas características clínicas relativas a la atención de una muestra de pacientes con patología arterial y con TVP ingresados en el servicio de cirugía vascular del hospital universitario Lucus Augusti de Lugo, tras la implantación de un modelo de asistencia multidisciplinar con internistas durante el año 2013. Los datos de los enfermos con TVP se compararon con los de controles históricos del año anterior. Por otra parte, se realizó una encuesta de satisfacción al personal sanitario del servicio de cirugía vascular sobre sus preferencias respecto al modelo de atención.

Resultados: se estudiaron 50 pacientes con patología arterial y 75 con TVP (25 casos y 50 controles). Entre los primeros, su edad fue 71.9 años (DE 14), 60\% hombres. Recibían una media de 8.2 fármacos (DE 4.4), su índice de Charlson fue 4.1 (DE 2.7) y el índice de Barthel 85.9 (DE 20). El 70\% tenía anemia al ingreso y el 86\% presentó alguna complicación durante el ingreso, con una mortalidad del $10 \%$.

Los pacientes con TVP atendidos por el modelo de asistencia compartida presentaron una mayor frecuencia de identificación de factores de riesgo de trombosis (76\% vs $38 \%$; $p=0.002)$, de ajuste correcto de dosis de HBPM al peso y función renal (92\% vs $18 \% ; p=0.0001$ ) y de registro completo de comorbilidad ( $100 \%$ vs $50 \% ; p=0.0001$ ), sin diferencias en la estancia media hospitalaria.

Tanto el personal facultativo como el de enfermería prefirieron el modelo de asistencia compartida con internistas respecto al estándar.

Conclusiones: los enfermos con patología arterial y venosa presentan una comorbilidad y riesgo de complicaciones que justifica la participación de internistas en su atención. El modelo de asistencia compartida puede mejorar la calidad de la asistencia prestada, con un elevado grado de satisfacción entre el personal del equipo quirúrgico.

Palabras clave: asistencia compartida, medicina interna, cirugía vascular

\section{Introducción}

El internista es, en atención a su polivalencia y capacidad de enfoque multidisciplinar, uno de los especialistas más requerido en las plantas quirúrgicas, especialmente cuando se trata de pacientes complejos, con múltiples problemas médicos añadidos al que motivó el ingreso ${ }^{1-3}$. Su concurso en la atención de estos enfermos garantiza la coordinación de todos los profesionales implicados en el caso y evita la

\begin{abstract}
Objective: to assess whether clinical comanagement between vascular surgeons and internists improves the quality of care provided to patients admitted to a vascular surgery service.
\end{abstract}

Material and method: several clinical features related to the clinical care provided to a sample of patients with arterial disease and DVT admitted to the vascular surgery at Lucus Augusti University Hospital of Lugo were analyzed, after implementation of a comanagement model of care involving internists during the year 2013. Data of patients with DVT were compared with historical controls of the year before. Moreover, we conducted a satisfaction survey to the medical staff and nursing of the vascular surgery service about their preferences regarding the model of clinical care.

Results: 50 patients with arterial disease and 75 with DVT ( 25 cases and 50 controls) were studied. Among the former, mean age was 71.9 years (SD 14), 60\% male. They received an average of 8.2 drugs (SD 4.4), the Charlson index was 4.1 (SD 2.7) and the Barthel index 85.9 (SD 20). 70\% had anemia at admission and 86\% experienced in-hospital complications, with a mortality of $10 \%$.

DVT patients attended by the comanagement model showed a higher frequency of identified risk factors for thrombosis $(76 \%$ vs $38 \%, p=0.002)$, proper adjustment of the dose of LMWH to weight and renal function (92\% vs $18 \%, p=0.0001)$ and complete record of comorbidity (100\% vs $50 \%$, $P=0.0001)$, with no differences in length of hospital stay.

Both medical staff and the nursing preferred the comanagement model with respect to the standard model.

Conclusions: patients with arterial and venous disease have a risk of complications and comorbidity that justify the participation of internists in their clinical care. The comanagement model can improve the quality of care provided, with a high degree of satisfaction among the staff of the surgical team.

Key words: comanagement, internal medicine, vascular surgery

confusión que genera en ocasiones la implicación simultánea de varios especialistas en el manejo de estos casos.

La colaboración clásica de los internistas en las plantas quirúrgicas se ha producido por medio de la interconsulta médica, trabajo realizado habitualmente a demanda en función de las complicaciones que presentan los enfermos ${ }^{1-4,6}$. En este modelo tradicional, los cirujanos y los anestesistas suelen limitarse a su área correspondiente, recurriendo al internista u otro 
especialista médico en función de la evolución del enfermo. Sin embargo, la interconsulta tiene el inconveniente de que su solicitud depende del médico responsable del paciente y de su habilidad para detectar problemas clínicos con los que frecuentemente no está familiarizado. Por otra parte, este sistema ocasiona con frecuencia fallos de comunicación y coordinación, que originan problemas de polifarmacia, duplicidad en la solicitud de pruebas complementarias y órdenes médicas a menudo confusas cuando no contradictorias. También se ha señalado que esta forma de actuación es poco eficaz y económicamente costosa ${ }^{6,7}$.

Una posible alternativa a este modelo es la asistencia compartida, mediante la formación de equipos multidisciplinares estables de trabajo conjunto entre clínicos, cirujanos y anestesistas, que deberían incluir también enfermeros, trabajadores sociales, fisioterapeutas, etc, encargados de procurar una atención integral al paciente, que atienda todos los aspectos clínicos relacionados con su proceso desde el mismo momento del ingreso, sin esperar a la aparición de complicaciones ${ }^{2,5,8,9}$. En este sistema, el internista se hace responsable de los problemas médicos desde el ingreso hasta el alta del enfermo, sirviendo de nexo entre el cirujano, el anestesista y otros especialistas cuando sea preciso, mejorando la comunicación entre todos los implicados en el proceso quirúrgico.

En España, la mayor parte de las experiencias publicadas respecto al apoyo de los clínicos en la atención de pacientes quirúrgicos se han centrado en los servicios de traumatología, y especialmente en los enfermos con fractura de cadera ${ }^{5,10-12}$. En los últimos años, se ha potenciado la creación de equipos de atención multidisciplinar, centrados en el paciente y siguiendo el modelo de arquitectura de procesos asistenciales integrados, que han conseguido una mejora en resultados beneficiosos de salud, traducidos en un elevado porcentaje de pacientes intervenidos precozmente, una reducida estancia media, una baja incidencia de complicaciones postquirúrgicas y una adecuada recuperación funcional, todo ello asociado a un ahorro importante en costes ${ }^{5,10,12}$ y a un elevado grado de satisfacción entre los profesionales implicados ${ }^{13,14}$.

En el ámbito de la cirugía no traumatológica también se han comunicado resultados favorables en cuanto a mejoras de calidad en la atención sanitaria ligada a la participación programada de los clínicos en la atención de pacientes quirúrgicos ${ }^{8,15}$. Sin embargo, en España únicamente se han publicado datos referentes a la actividad general relacionada con el trabajo a demanda mediante realización de interconsultas médicas ${ }^{3,4}$.

El caso concreto de los servicios de cirugía vascular supone un reto especial para la participación del internista en la atención de enfermos quirúrgicos. En su vertiente de patología arterial, se trata de pacientes con aterosclerosis evolucionada, una prevalencia de enfermedad coronaria que puede alcanzar el $90 \%$ si se realizan estudios angiográficos y una mortalidad que triplica la de los pacientes sin arteriopatia ${ }^{16}$, en los que además es frecuente encontrar otros factores de comorbilidad como son la diabetes, infecciones graves e insuficiencia renal. Además, los procedimientos quirúrgicos que se realizan son de un grado elevado de complejidad y de riesgo de complicaciones (grado IV de la NICE ${ }^{17}$ ).

Por otra parte, existen muchos centros hospitalarios, como es el caso del Hospital Universitario Lucus Augusti, donde los enfermos con patología venosa de miembros inferiores ingresan de primera intención en los servicios de cirugía vascular. La incidencia global de TVP en la población general se sitúa alrededor de 5 casos por 10.000 pacientes/año y presenta un incremento progresivo con la edad, lo que aumenta la carga de enfermedades crónicas coexistentes en estos enfermos ${ }^{18}$. En nuestro país, según datos del Ministerio de Sanidad y Consumo, la enfermedad tromboembólica venosa supuso el $0,8 \%$ de todas las altas hospitalarias entre los años 1999 y 2005. Por otra parte, se ha calculado que los gastos asociados a la enfermedad tromboembólica venosa son de 76 millones de euros anuales, de los cuales el 80\% corresponde a los gastos hospitalarios ${ }^{19}$.

La valoración clínica indicada en un paciente con TVP incluye una completa historia clínica con análisis de comorbilidad y de factores de riesgo de trombosis venosa, la elección de tratamiento teniendo en cuenta factores como el riesgo hemorrágico, el peso o la función renal, la determinación del riesgo de desarrollar y en su caso la presencia de un embolismo pulmonar, una adecuada elección de pruebas complementarias añadidas a la diagnóstica inicial de TVP y un seguimiento clínico en el tiempo que asegure tanto la adherencia al tratamiento como su correcta duración y la detección precoz de complicaciones ${ }^{20,21}$. Por otra parte, es bien conocida la posible presencia de un cáncer subyacente como factor de riesgo de TVP, especialmente en pacientes de edad avanzada y con trombosis idiopática ${ }^{21,22}$. Sin embargo, no está claro si es coste-efectiva la actitud de realizar un estudio exhaustivo diagnóstico de búsqueda de neoplasia tras el diagnóstico de TVP, puesto que los estudios realizados no muestran diferencias en la mortalidad ligada a un diagnóstico precoz ${ }^{23,24}$. No obstante, sí se recomienda una valoración clínica dirigida por la historia clínica y pruebas complementarias básicas, unida a un seguimiento activo prolongado ${ }^{25}$.

El objetivo de este trabajo es valorar si la asistencia clínica compartida entre cirujanos vasculares e internistas mejora la calidad de la atención médica prestada a pacientes ingresados en el servicio de Cirugía Vascular, tanto con patología venosa como arterial, y si la formación de un equipo multidisciplinar en este contexto mejora el nivel de satisfacción del personal sanitario facultativo y no facultativo respecto al modelo tradicional de atención. 


\section{Método}

\section{Ámbito de trabajo}

El Hospital Universitario Lucus Augusti de Lugo es un centro docente de $2^{\circ}$ nivel, que dispone de 869 camas, con las que se cubre la asistencia sanitaria de una población de 221.441 personas, de ámbito mixto, rural y urbano. El servicio de cirugía vascular y Angiología tiene asignadas 24 camas y está integrado por 10 cirujanos vasculares, 4 residentes de cirugía vascular y 15 diplomados en enfemería. Atiende a la población con patología vascular arterial y venosa del área sanitaria integrada de gerencia única de la provincia de Lugo, la cual asciende a 348.902 personas.

\section{Programa de asistencia compartida (multidisciplinar)}

Desde el mes de febrero de 2013, 2 internistas se adscribieron al servicio de cirugía vascular. Por razones operativas y de sobrecarga asistencial, su labor se centró en la atención en todos los enfermos con TVP y en aquellos con patología arterial que a juicio del especialista de cirugía vascular precisasen una evaluación clínica por parte de un especialista médico. El modelo de atención siguió el principio general de actuación en el que la responsabilidad sobre el paciente desde su llegada a Urgencias hasta el alta es compartida entre el internista y el cirujano vascular, de forma reglada, diaria y adaptada a la situación clínica que sea preciso atender, informando ambos al enfermo y/o a su familia en caso de autorización de éste 0 incapacidad. Las tareas realizadas por el internista fueron similares a la que se realizan habitualmente en una planta de Medicina Interna, con independencia total en la solicitud de pruebas complementarias. El nuevo modelo de asistencia supuso una reorganización de la actividad asistencial del servicio de medicina interna, ajustando la carga de trabajo para que 2 internistas pudieran atender la nueva tarea, sin implicar un aumento en el número de facultativos.

Los pacientes con previsión de intervención quirúrgica o revascularización endovascular, en su mayoría con patología arterial, fueron evaluados tras su ingreso con el fin de mejorar en lo posible su estado y corregir los factores de comorbilidad con vistas a la cirugía. La valoración preanestésica fue solicitada por el cirujano vascular, aunque la decisión sobre la idoneidad del momento para la intervención fue tomada conjuntamente por Cirugía Vascular, Anestesiología y Medicina Interna. El internista decidió sobre la necesidad de consultar a otras especialidades médicas cuando se estimó preciso y no intervino en la indicación quirúrgica. El internista continuó con el seguimiento diario de su evolución clínica y tratamiento de las eventuales complicaciones médicas hasta el momento del alta.

En aquellos enfermos con TVP se realizó una historia clínica completa con valoración de factores de riesgo de trombosis venosa y de comorbilidad. Se valoró conjuntamente la idonei- dad de la solicitud de pruebas complementarias adicionales y las opciones de tratamiento anticoagulante. Se realizó control clínico diario y de las posibles complicaciones hasta el momento del alta. Posteriormente, se programó el seguimiento tanto en la consulta de medicina interna (monográfica de enfermedad tromboembólica venosa) como en la de cirugía vascular.

Se realizó una reunión semanal entre los integrantes del equipo, con el fin de programar la actividad asistencial, coordinar las altas hospitalarias y potenciar la interrelación entre los miembros del grupo. Cuando fue necesario, el internista completó los informes de alta de cirugía vascular.

\section{Patología arterial}

Se analizaron las características de todos los pacientes ingresados con patología arterial valorados por el modelo de asistencia compartida (con internistas), entre el 1 de marzo y el 30 de abril de 2013. Se registraron las siguientes variables: edad, sexo, número de fármacos domiciliarios, índice de comorbilidad de Charlson, clasificación ASA (American Society of Anestesiologists), índice de dependencia de Barthel, filtrado glomerular estimado (MDRD), Hemoglobina (al ingreso, en gr/ dl), Albúmina (al ingreso, en mg/dl), motivo de ingreso, realización de intervención quirúrgica y/o tratamiento endovascular, estancia hospitalaria y mortalidad. Para el análisis de las complicaciones desarrolladas durante el ingreso, estas se agruparon en: cardiovasculares (insuficiencia cardiaca, taquiarritmias, síndrome coronario agudo, accidente cerebrovascular agudo, enfermedad tromboembólica venosa), neurológicas (síndrome confusional agudo), infecciosas (infección respiratoria de vías bajas con o sin neumonía radiológica, infección urinaria, bacteriemia), trastorno eletrolítico-metabólico (descompensación de diabetes, insuficiencia renal aguda, trastorno iónico que requiere tratamiento) y otras complicaciones (escaras, íleo intestinal, hemorragia digestiva, reintervención quirúrgica, dehiscencia de sutura).

\section{Patología venosa (TVP)}

Se analizaron todos los pacientes con TVP evaluados por el modelo de asistencia compartida (con internistas) entre el 1 de febrero y el 30 de agosto de 2013. Por cada caso se seleccionaron 2 controles valorados por el modelo de asistencia tradicional (sin internistas), ajustados por edad y sexo, entre los pacientes con TVP ingresados en el servicio de cirugía vascular durante el año 2012. Para cada episodio se registraron las siguientes variables: edad, sexo, localización de la TVP, factores de riesgo de tromboembolismo venoso identificados y tipo (estrógenos, embarazo, puerperio, cáncer, inmovilización prologada, tiempo encamado superior a 5 días, cirugía en los 3 meses previos, varices, trombofilia, TVP previa), anotación del peso de los pacientes en la historia clínica, ajuste de la dosis de heparina de bajo peso molecular (HBPM) administrada ajustada al peso y a la función renal, registro completo 
de comorbilidad, número de diagnósticos de enfermedades no conocidas previamente realizados durante el ingreso y tipo, estancia hospitalaria, mortalidad y seguimiento clínico ambulatorio especializado.

\section{Encuesta de satisfacción}

En el mes de septiembre de 2013 se entregó una encuesta confidencial al personal facultativo y de enfermería del servicio de cirugía vascular acerca de su nivel de satisfacción respecto al modelo de trabajo multidisciplinar con internistas, de asistencia compartida, comparado con el tradicional previo a la implantación de este modelo. La encuesta fue autoadministrada, confidencial y anónima, estableciendo un plazo máximo de respuesta de 48 horas. Las preguntas de la encuesta (adaptado de Huddleston JM et al ${ }^{17}$ ) fueron de tipo cerrado, con 5 posibles respuestas a las que se asignó un valor ordinal: 1. Nivel de comunicación entre internistas y cirujanos vasculares; 2 Órdenes médicas breves y específicas; 3. Facilidad de localización del internista; 4. Calidad de atención médica prestada; 5. Delimitación de la responsabilidad sobre el enfermo; 6 . Coordinación del personal facultativo; 7. Coordinación para la programación del alta hospitalaria; 8. Valoración de las necesidades postoperatorias del enfermo; 9. Celeridad de atención ante un problema postoperatorio; 10. Conocimiento del internista de los cuidados quirúrgicos y de enfermería postoperatorios. En todos los apartados se ofrecían 5 posibles respuestas: tradicional mucho mejor (1 punto), tradicional algo mejor (2 puntos), neutral (3 puntos), multidisciplinar algo mejor (4 puntos), multidisciplinar mucho mejor (5 puntos).

\section{Análisis estadístico}

Las bases de datos fueron diseñadas mediante el programa Excel 2007 y los datos fueron analizados con el programa estadístico SPSS15. En el análisis estadístico se utilizó el test de la $x^{2} 0$ el test exacto de Fisher para evaluar la asociación entre dos variables cualitativas. Para la comparación de variables cuantitativas entre grupos se utilizó el test de la t de Student o el test de Mann-Whitney, previa comprobación de la normalidad de las variables con el test de Kolmogorov-Smirnov.

\section{Confidencialidad}

La confidencialidad de datos ha sido tratada de acuerdo con la ley orgánica de protección de datos de carácter personal 15/1999.

\section{Resultados}

\section{Patología arterial}

Se estudiaron las características de 50 pacientes ingresados por patología arterial. La edad media fue 71.9 años (DE 14), el $60 \%$ hombres. El motivo de ingreso fue arteriopatía periférica de miembros inferiores establecida o complicaciones derivadas de la misma en 40 casos (80\%), embolia arterial en $3(6 \%)$, aneurisma de aorta abdominal en $3(6 \%)$, endarterectomia carotidea en $3(6 \%)$ y en 1 caso estenosis de arteria subclavia. Todos los enfermos recibían medicación crónica domiciliaria, con un promedio de 8.2 fármacos (DE 4.4). De ellos, 43 (86\%) tomaban 4 o más fármacos y $15(30 \%)$, más de 10 . Treinta y cinco enfermos $(70 \%)$ presentaban anemia al ingreso (hemoglobina $<13 \mathrm{gr} / \mathrm{dl}$ en hombres y $<12 \mathrm{gr} / \mathrm{dl}$ en mujeres), y de ellos el 48\% tenían una hemoglobina inferior a $10 \mathrm{gr} / \mathrm{dl}$. El filtrado glomerular medio estimado mediante la ecuación MDRD-4 IDMS (ml/min/1,73 m²) fue 74.6 (DE 40.2) e inferior a 60 en el $38 \%$ de los casos.

Los resultados de la estratificación de riesgo anestésico según la clasificación ASA, del índice de comorbilidad de Charlson (no ajustado por edad) y del índice de dependencia funcional de Barthel se muestran en la tabla 1.

Tabla 1. Estratificación de comorbilidad (ASA, índice de Charlson) y dependencia (índice de Barthel) en pacientes ingresados por patología arterial

\begin{tabular}{|lc|}
\hline \multicolumn{1}{|l|}{ ASA } & $\mathrm{n}=50(\%)$ \\
\hline I-II & $14(28)$ \\
III-IV & $36(72)$ \\
Barthel & \\
Barthel & $85.9(20)$ \\
$80-100$ (leve) & \\
$60-79$ (moderada) & $36(72)$ \\
$46-59$ (grave) & $7(14)$ \\
$<45$ (severa) & $6(12)$ \\
Charlson* & $1(2)$ \\
Charlson & $4.1(2.7)$ \\
$<4$ (bajo) & \\
$4-5$ (moderado) & $26(52)$ \\
-7 (alto) & $10(20)$ \\
$>7$ (muy alto) & $8(16)$ \\
& $6(12)$ \\
\hline
\end{tabular}

Veintinueve enfermos (58\%) precisaron intervención quirúrgica y 15 (30\%) tratamiento intervencionista endovascular. Cuarenta y tres pacientes (86\%) sufrieron algún tipo de complicación durante el ingreso, de las cuales fueron infecciosas en 28 casos (56\%), hidroelectrolítica-metabólicas en 20 (40\%), cardiovasculares en 17 (34\%), neurológicas en 8 (16\%) y quirúrgicas/otras en 26 (52\%). La estancia media fue 27.9 días (DE 23.9). Precisaron traslado a medicina interna 5 pacientes (10\%). Fallecieron 5 enfermos (10\%). 


\section{Patología venosa (TVP)}

Se analizaron las características de 25 pacientes (12 hombres y 13 mujeres) con TVP valorados según el modelo de asistencia compartida (casos). Por cada caso se seleccionaron 2 controles históricos del año anterior a la implantación del modelo, valorados mediante el sistema tradicional, ajustados por edad y sexo.

En global, teniendo en cuenta los 75 enfermos, la edad media fue 70.4 (DE 13.2), el 48\% hombres. La localización más frecuente de la TVP fue femoropoplítea en 35 casos (46.6\%), poplítea en 27 (36\%), femoral en 8 (10.6\%), ileofemoral en $4(5.3 \%)$ e ileofemoropoplítea en 1 (1.3\%). Se identificó durante el ingreso al menos 1 factor de riesgo de TVP en 37 pacientes (49.3\%): inmovilización previa en 20 (26.7\%), varices en 12 (13.3\%), cáncer en 10 (13.3\%), TVP previa en 5 (6.7\%), cirugía o ingreso reciente en 3 (4\%) y trombofilia en $1(1.3 \%)$. La estancia media hospitalaria fue 4.7 dias (DE 4.6), la mortalidad al alta hospitalaria 1.3\% y a los 3 meses $4 \%$. Todos los enfermos fueron tratados con HBPM durante el ingreso hospitalario.

Los datos referentes a la comparación de casos (pacientes valorados según el modelo de asistencia multidisciplinar) y controles (pacientes valorados según el modelo estándar), se muestran el tabla 2. Los pacientes valorados mediante el sistema de asistencia multidisciplinar presentaron una mayor frecuencia de registro de comorbilidad y del peso en la historia clínica, así como de ajuste de la dosis de HBPM según este parámetro y el filtrado glomerular. También se identificaron con más frecuencia factores de riesgo de TVP en los casos que en los controles. Por otra parte, su valoración durante

Tabla 2. Comparación entre el modelo de asistencia compartida (multidisciplinar) y el modelo estándar aplicado a pacientes con TVP

\begin{tabular}{|c|c|c|c|}
\hline & $\begin{array}{c}\text { Modelo } \\
\text { multidisciplinar } \\
n=25(\%)\end{array}$ & $\begin{array}{l}\text { Modelo estándar } \\
\text { n=50 (\%) }\end{array}$ & $p$ \\
\hline Edad* $^{*}$ & 70.7 (12.3) & $70.2(13.8)$ & ns \\
\hline Hombres & $12(48)$ & $24(48)$ & ns \\
\hline $\begin{array}{l}\text { Identificación de factores } \\
\text { de riesgo }\end{array}$ & $19(76)$ & $19(38)$ & 0.002 \\
\hline $\begin{array}{l}\text { Registro del peso del } \\
\text { paciente }\end{array}$ & $23(92)$ & $10(20)$ & 0.0001 \\
\hline Dosis de HBPM ajustada ${ }^{a}$ & $23(92)$ & $9(18)$ & 0.0001 \\
\hline Registro de comorbilidad & $25(100)$ & $25(50)$ & 0.0001 \\
\hline Diagnósticos "de novo" $\beta$ & $12(48)$ & $5(10)$ & 0.0001 \\
\hline Traslado a Ml & $4(16 \%)$ & $0(0)$ & 0.004 \\
\hline Estancia hospitalaria* & $4.8(3)$ & $4.6(5.2)$ & ns \\
\hline $\begin{array}{l}\text { Seguimiento ambulatorio } \\
\text { por Ml }\end{array}$ & $24(96)$ & $32(64)$ & 0.003 \\
\hline
\end{tabular}

*Media (DE)

aDosis de HBPM ajustada por el peso del paciente y su filtrado glomerular

ßDiagnósticos de enfermedades no conocidas previamente al ingreso, diferentes de la TVP: 1 caso de absceso renal, anemia ferropénica, insuficiencia renal crónica, bursitis rotuliana, cáncer de próstata, nódulos pulmonraes, esofagitis, flutter permanente, gota, prostatitis aguda, policitemia vera, esteatosis hepática; 2 casos de HTA; 2 casos de trombofilia por factor $V$ Leiden. el ingreso por internistas se asoció de forma significativa a la realización de diagnósticos de patologías no conocidas previamente (diferentes a TEP), de interés evidente en su manejo clínico. Se trasladaron a la planta de hospitalización de medicina interna 4 pacientes en el grupo de los casos, por los siguientes motivos: absceso renal, anemia, nódulos pulmonares, TEP. Falleció 1 paciente durante el ingreso en el grupo control y ninguno en el de asistencia multidisciplinar. La mortalidad a los 3 meses de seguimiento fue de 1 enfermo en el grupo de asistencia con internistas y 2 en el grupo control.

\section{Encuesta de satisfacción}

De un total de 14 facultativos (10 especialistas en cirugía vascular y 4 residentes) y 15 diplomados en enfermería respondieron 11 y 12 profesionales, respectivamente. Todos ellos habían trabajado con ambos modelos de atención.

Tanto el personal de enfermería como el facultativo prefirieron el modelo multidisciplinar de atención. En general, las opiniones del personal facultativo fueron más favorables hacia el modelo de asistencia compartida que las del personal de enfermería [42,27 puntos totales sobre 50 posibles (DE 3,69) vs 35,27 (DE 4,4); $p<0,001)$ ]. Los valores medios obtenidos en cada pregunta, comparados entre ambos grupos profesionales se muestran en la tabla 3.

Tabla 3. Encuesta de satisfacción al personal del servicio de cirugía vascular sobre un modelo de asistencia multidisciplinar con internistas

\begin{tabular}{|c|c|c|c|c|}
\hline & & $\begin{array}{l}\text { Facultativos } \\
\qquad(\mathrm{n}=11)\end{array}$ & $\begin{array}{c}\text { DUE } \\
(n=12)\end{array}$ & $p$ \\
\hline 1 & $\begin{array}{l}\text { Nivel de comunicación } \\
\text { entre cirujanos e } \\
\text { internistas }\end{array}$ & $4,5(0.5)$ & $4,1(0,79)$ & NS \\
\hline 2 & $\begin{array}{l}\text { Ordenes medicas breves y } \\
\text { especificas }\end{array}$ & $4,4(0,5)$ & $3,1(1,4)$ & 0,012 \\
\hline 3 & $\begin{array}{l}\text { Facilidad de localización } \\
\text { del internista }\end{array}$ & $4,1(0,9)$ & $4,3(0,6)$ & NS \\
\hline 4 & $\begin{array}{l}\text { Calidad de atención } \\
\text { medica prestada }\end{array}$ & $4,4(0,6)$ & $4,3(0,4)$ & NS \\
\hline 5 & $\begin{array}{l}\text { Delimitación de la } \\
\text { responsabilidad sobre el } \\
\text { enfermo }\end{array}$ & $4,1(0,7)$ & $2,6(0,8)$ & 0,0001 \\
\hline 6 & $\begin{array}{l}\text { Coordinación del personal } \\
\text { facultativo }\end{array}$ & $4,4(0,5)$ & $3,3(0,9)$ & 0,003 \\
\hline 7 & $\begin{array}{l}\text { Coordinación para la } \\
\text { programación del alta } \\
\text { hospitalaria }\end{array}$ & $4(0,8)$ & $3,1(0,9)$ & 0,02 \\
\hline 8 & $\begin{array}{l}\text { Valoración de las } \\
\text { necesidades post- } \\
\text { operatorias del enfermo }\end{array}$ & $4,2(0,6)$ & 3,7 (1) & NS \\
\hline 9 & $\begin{array}{l}\text { Celeridad de atención } \\
\text { ante un problema post- } \\
\text { operatorio }\end{array}$ & $4,5(0,5)$ & $4(0,7)$ & NS \\
\hline 10 & $\begin{array}{l}\text { Conocimiento del } \\
\text { internista de los cuidados } \\
\text { post-operatorios }\end{array}$ & $3,8(0,6)$ & $3,4(0,9)$ & NS \\
\hline
\end{tabular}

DUE: diplomados universitarios en enfermería Valores expresados como media (DE) 


\section{Discusión}

El presente estudio muestra que los pacientes ingresados en un servicio de cirugía vascular presentan un perfil clínico cuyas características aconsejan la intervención conjunta de especialistas médicos y quirúrgicos en su manejo. Esta intervención, bajo la forma de un modelo de asistencia compartida con internistas, multidisciplinar, es capaz de mejorar la calidad de la asistencia médica ofrecida a los enfermos, además con un elevado grado de satisfacción entre el personal sanitario del equipo quirúrgico.

\section{Patología arterial}

La enfermedad arterial periférica es una manifestación secundaria a una arterosclerosis sistémica y está asociada a un incremento del riesgo de morbilidad y mortalidad cardiovascular ${ }^{15}$. El registro internacional REACH (Reduction of Atherothrombosis for Continued Health) evidenció que los pacientes diagnosticados de esta entidad tienen una mayor tasa de episodios cardiovasculares que los afectados por una enfermedad coronaria o cardiovascular ${ }^{26}$. Por otra parte, hasta el $25 \%$ de estos enfermos son diabéticos y aproximadamente la mitad de los pacientes diabéticos mayores de 70 años presenta arteriopatía periférica ${ }^{27}$. También se ha descrito una marcada relación entre la existencia de insuficiencia renal crónica y arteriopatía periférica, que se presentará de forma más agresiva y con peor pronóstico en su presencia ${ }^{28}$.

Todas estas circunstancias explican los elevados índices de comorbilidad (Charlson), moderado o más elevado en la mitad de los casos, de más trascendencia teniendo en cuenta que no se empleó el modelo ajustado por la edad, como en otros estudios realizados en pacientes quirúrgicos ${ }^{12}$. En el mismo contexto se deben interpretar los resultados de la estratificación de riesgo anestésico ASA, en grupo II 0 III en más de los 2/3 de los enfermos.

La polifarmacia acompaña con frecuencia a estos enfermos, como muestran nuestros datos (más de 8 fármacos de media por enfermo), con el riesgo consiguiente de aparición de efectos secundarios durante el periodo perioperatorio, como resultado de los cambios fisiológicos que se producen en su absorción y otros parámetros como consecuencia de la cirugía y de la suspensión de la medicación oral2 ${ }^{29}$. En este sentido, el ingreso hospitalario debe servir para detectar y evitar la administración de medicamentos potencialmente inadecuados, circunstancia que se produce con más frecuencia cuando son múltiples especialistas los que prescriben la medicación ${ }^{30}$.

El desarrollo de complicaciones hospitalarias médicas o quirúrgicas en estos enfermos resultó frecuente (86\%), como consecuencia de la conjunción de todos los factores comentados anteriormente, relativos a su comorbilidad y a la complejidad de la enfermedad que motivó el ingreso, con una mortalidad hospitalaria que alcanzó el 10\% en la serie. Como se detalla en el estudio, las complicaciones médicas no difieren mucho de las patologías atendidas habitualmente en las plantas de hospitalización de medicina interna y coinciden con las detectadas en otros trabajos sobre consultas de servicios médicos en servicios quirúrgicos 33,412 .

\section{Patología venosa (TVP)}

El modelo de asistencia compartida con internistas recogió con más frecuencia datos en la historia clínica referentes a la comorbilidad de los pacientes y a su peso. También identificó con más precisión la presencia de factores de riesgo de TVP. No es sorprendente que, puesto que la labor del internista se realiza fundamentalmente a través de una eficaz valoración clínica, los datos muestren estos resultados. En todo caso, una adecuada valoración de estas variables, acerca la práctica clínica habitual en pacientes con TVP a unos estándares de calidad $^{20,21}$. En el mismo sentido, la inclusión del punto de vista y quehacer habitual del clínico estimula la realización de diagnósticos que, aunque no directamente relacionados con la TVP, serán de relevancia en el manejo general del enfermo, como atestigua este trabajo, en línea con otros estudios sobre interconsultas en pacientes quirúrgicos ${ }^{3,4}$

Una completa historia clínica que recoja la existencia de factores de riesgo de TVP, puede limitar la petición de estudios de trombofilia hereditaria que no estarían indicados o de cribado de neoplasia innecesarios. En términos generales, la realización de estudios de este tipo debe estar dirigida por una adecuada valoración clínica global y actualmente se tiende a matizar su indicación ${ }^{23-25,31}$. En este sentido, la práctica habitual en nuestro centro, antes de la implantación del nuevo modelo de asistencia mediante interconsulta y ahora de forma programada, era y es la valoración por parte del especialista de medicina interna de los pacientes con ETV idiopática y sospecha de neoplasia, de acuerdo con un protocolo establecido ${ }^{32}$. La evidencia actual orienta a que variables como la existencia de un cáncer asociado, el riesgo de hemorragia, los antecedentes familiares o la presencia de un factor desencadenante de trombosis, son más importantes que el hallazgo de un defecto trombofílico para el manejo de la enfermedad tromboembólica ${ }^{31}$.

Por otra parte, en el tratamiento de la enfermedad tromboembólica venosa con HBPM, es necesario el ajuste de la dosis en función del peso del enfermo y de su filtrado glomerular, con el objetivo de asegurar un adecuado efecto anticoagulante y minimizar el riesgo de efectos secundarios hemorrágicos derivados de la presencia de grados avanzados de insuficiencia renal ${ }^{33,34}$. El hecho de que el registro del peso o la comprobación de la corrección de la dosis de HBPM ajustada a estas variables, fuera menos frecuente en el modelo tradicional de asistencia, no asegura que los pacientes estuvieran mal tratados, pero es evidente que su registro acerca la práctica clínica a los estándares recomendados ${ }^{31}$. 


\section{Encuesta de satisfacción}

Tanto el personal facultativo como el de enfermería prefirieron el modelo multidisciplinar de asistencia compartida con internistas integrados en el equipo de atención médica del servicio de cirugía vascular, sobre el modelo tradicional, con resultados similares a los obtenidos en otras encuestas realizadas en servicios de traumatología y ortopedia ${ }^{13,14}$.

Los cirujanos tienden a valorar más favorablemente el modelo multidisciplinar $^{14}$, probablemente debido a que son los profesionales más directamente afectados por las mejoras que se derivan del mismo. Como ya se ha comentado, la asistencia médica de pacientes quirúrgicos es compleja y precisa cada vez con más frecuencia de la incorporación de habilidades clínicas que están en ocasiones alejadas de las empleadas habitualmente por los cirujanos. Por otra parte, en el modelo tradicional, la participación de los clínicos es requerida mediante interconsulta, frecuentemente múltiple en función de las diversas patologías y/o complicaciones que presenta el enfermo. Esto hace que la labor de localización y coordinación de los especialistas implicados sea ingrata, y que la rapidez de respuesta ante un problema urgente pueda verse comprometida. Así, los facultativos ponderaron el modelo de asistencia compartida especialmente en los aspectos relativos al nivel de comunicación entre internistas y cirujanos, su coordinación y la celeridad de actuación ante un problema postoperatorio. El personal de enfermería, aunque favorable al modelo multidisciplinar, percibe menos ventajas que los facultativos. De hecho, es posible que la presencia continuada de un clínico en el servicio, aumente y/o modifique las órdenes de tratamiento que se realizan en el modelo tradicional (pregunta $n^{0} 2$ ), con el consiguiente aumento de la carga de trabajo. Por otra parte, si bien los cirujanos se sienten apoyados por el internista en cuanto a la delimitación de la responsabilidad sobre el enfermo, las enfermeras no lo valoran de la misma manera (pregunta n5). Esta visión aparentemente contradictoria cambiaría probablemente si el modelo aclarase mejor una cuestión clave en este sentido: "¿a quién aviso si el paciente empeora, al cirujano 0 al internista?", cuya respuesta implica no solo al equipo de asistencia ordinario sino también al de guardia y posiblemente a la dirección médica. No obstante, tanto el equipo quirúrgico como el de enfermería coinciden en valorar positivamente los aspectos claves del modelo de asistencia compartida: aumenta la facilidad de localización del clínico, la celeridad de actuación ante un problema postoperatorio y, más importante, aumenta la calidad de la asistencia prestada. Finalmente, ambos grupos opinan que los conocimientos del internista respecto a los cuidados quirúrgicos y de enfermería postoperatorios no han mejorado mucho con el nuevo modelo, un aspecto que es de esperar mejore con el tiempo.

En este estudio, como en otros ${ }^{5,8,10,13}$, el desarrollo del modelo multidisciplinar ha estado ligado a la formación de un equi- po basado en la continuidad en el tiempo de los internistas destinados al mismo. Esta actitud favorece una coordinación adecuada de los profesionales implicados mediante el establecimiento de relaciones interpersonales que mejoran el nivel de comunicación y las posibilidades de trabajo en equipo.

\section{Calidad asistencial}

El objetivo de la gestión clínica, y por extensión, de una reforma en el modelo de atención es el de mejorar la calidad de los servicios, salvaguardar unos estándares estrictos de asistencia y crear un entorno que facilitará la excelencia de la asistencia sanitaria ${ }^{35}$. La cuestión central en este caso es el aumento de resultados del paciente (estado de salud) obtenidos al menor coste.

En este trabajo, la producción de servicios se ha medido a través de indicadores de proceso (volumen, adecuación a estándares clínicos, diagnósticos, satisfacción), que si bien son Ios más fáciles y frecuentes de obtener, no miden realmente la repercusión sobre la salud del paciente, como harían los indicadores de resultado, más complejos de construir. No se recogieron datos de consumo de recursos, más allá de la significación que tiene la estancia media, por lo que no podemos valorar el coste estricto del nuevo modelo. En éste, mientras que por un lado se adecua el consumo de recursos, la evaluación completa de los enfermos por parte de un clínico puede descubrir nuevos problemas que obliguen a realizar otras pruebas diagnosticas o terapéuticas, como queda de manifiesto en los resultados del estudio. No obstante, el objetivo de una reforma de gestión clínica es la de aumentar la eficiencia, lo que aplicado a un servicio o a una unidad de gestión clínica, se define como la consecución de una adecuada relación entre el coste y la producción de servicios ${ }^{36}$. En este sentido, el nuevo modelo de asistencia ha mejorado la adecuación de la práctica clínica a los estándares de calidad, con un elevado grado de satisfacción entre el personal sanitario del equipo quirúrgico, a la vez que no ha supuesto un aumento en el coste de recursos humanos ni del tiempo de hospitalización de los pacientes. Por estos motivos, se puede afirmar que ha tenido una repercusión favorable en la calidad de la asistencia prestada.

\section{Limitaciones}

Este estudio presenta varias limitaciones. El tamaño muestral es pequeño, el análisis es retrospectivo y, en lo relativo a la TVP compara cohortes históricas, valoradas en diferentes periodos. Sin embargo, al menos en cuanto a la dotación de recursos humanos y estructurales de ambos periodos, la única diferencia fue la presencia constante de internistas en el modelo de asistencia compartida.

Por otra parte, hay que señalar un posible sesgo de intervención, en cuanto a que el modelo multidisciplinar está diseñado precisamente para mejorar aquellos variables en las que sale 
beneficiado (valoración clínica eficaz y completa). Sin embargo, esta circunstancia no le resta al modelo una mayor capacidad de acercamiento a los estándares de calidad en la asistencia.

En cuanto a las encuestas de satisfacción, estas no incluyeron la opinión de los enfermos, que son los auténticos receptores de las mejoras realizadas en la calidad de la atención sanitaria.

\section{Conclusión}

La implicación de los clínicos en la atención de pacientes quirúrgicos es un fenómeno reciente en nuestro país, que aumentará con seguridad en los próximos años. Los internistas son, en atención a sus características de médicos hospitalarios generalistas, probablemente los especialistas idóneos para liderar esta reforma. El modelo de asistencia tradicional, en el que el cirujano solicita interconsultas a diferentes especialistas médicos en función de las complicaciones o características de sus enfermos, será posiblemente superado en un futuro cercano por la formación de equipos multidisciplinares integrados por clínicos y cirujanos donde el modelo de asistencia será compartido. Ya existían experiencias favorables en este sentido en España, especialmente en servicios de traumatología, y este trabajo aporta evidencias similares en un servicio de cirugía vascular, con una repercusión favorable en la calidad de la asistencia clínica y servicios prestados, además de con un elevado grado de satisfacción entre el personal del equipo quirúrgico.

\section{Bibliografía}

1. Charlson ME, Cohen RP, Sears CL. General medicine consultation. Lessons from a clinical service. Am J Med 1983; 75: 121-28.

2. Mollema R, Berger $P$, Girbes AR. The value of peri-operative consultation on a general surgical ward by the internist. Neth J Med. 2000; 56(1):7-11

3. Monte Secades R, Rabuñal Rey R, Rigueiro Veloso MT, García Pais MJ, Casariego Vales E, Guerrero Lombardía J. Papel del internista como consultor de servicios quirúrgicos. Rev Clin Esp 2004; 204: 345-50.

4. Montero Ruiz E, López Álvarez J, Hernández Ahijado C. Estudio de las interconsultas solicitadas a un servicio de Medicina Interna. Rev Clin Esp 2004; 204: 507-10.

5. Montero Ruiz E, Hernández Ahijado C, López Álvarez J. Efecto de la adscripción de un internista a un servicio quirúrgico. Med Clin (Barc) 2005; 124: 332-35.

6. Goldman L, Lee T, Rudd P. Ten commandments for effective consultations. Arch Intern Med 1983; 143: 1753-55

7. Klein LE, Levine DM, Moore D, Kirby SM. The preoperative consultation. Response to internists'recommendations. Arch Intern Med 1983; 1434: 743-4

8. Macpherson DS, Parenti C, Nee J, Petzel RA, Ward H. An internist joins the surgery service: does comanagement make the difference? J Gen Intern Med 1994; 9: 440-44.

9. Phy MP, Bañes DJ, Melton J, Long KH, Schleck CD, Larson DR, Huddleston PM, Huddleston JM. Effects of a hospitalist model on elderly patients with hip fracture. Arch Intern Med 2005; 165: 796-80

10. Fernández-Moyano A, Fernández-Ojeda R, Ruiz-Romero V, García-Benítez B, Palmero-Palmero C, Aparicio-Santos R Programa de atención integral a pacientes mayores de 65 años con fractura de cadera. Rev Clin Esp. 2013 http://dx.doi.org/ 10.1016/j. rce.2013.01.011.

11. Vidán M, Serra JA, Moreno C, Riquelme G, Ortiz J. Efficacy of a comprehensive geriatric intervention in older patients hospitalized for hip fracture: a randomized, controlled trial. J Am Geriatr Soc. 2005; 53(9): 1476-82.

12. Monte-Secades R, Peña-Zemsch $M$, Rabuñal-Rey R, Bal-Alvaredo M, Pazos-Ferro A, Mateos-Colino A. Factores de riesgo para el desarrollo de complicaciones en pacientes con fractura de cadera. Rev Calid Asist 2011; 26:76-82.

13. Huddleston JM, Long KH, Naessens JM, Vanness D, Larson D, Trousdale R, Plevak M, Cabanela M, Ilstrup D, Wachter RM. Medical and surgical comanagement afte elective hip and knee arthroplasty: a randomized, controlled trial. Ann Intern Med. 2004; 141: 28-38

14. Monte Secades R, Rabuñal Rey R, Peña Zemsch M, Bal Alvaredo M. Adscripción de un internista a un servicio de cirugía ortopédica y traumatología: encuesta de satisfacción. Rev Clin Esp. 2011; 21: 267-8.

15. Sharma G, Kuo YF, Freeman J, Zhang DD, Goodwin JS. Comanagement of hospitalized surgical patients by medicine physicians in the United States. Arch Intern Med. 2010; 170: 363-8

16. Mohler ER 3rd Peripheral arterial disease: identification and implications. Arch Intern Med. 2003; 163: 2306-14

17. Reynolds TM. National Institute for Health and Clinical Excellence. National Institute for Health and Clinical Excellence guidelines on preoperative tests: the use of Soutine preoperative tests for elective surgery. Ann Clin Biochem 2006; 43: 13-6.

18. Fowkes FJ, Price JF, Fowkes FG. Incidence of diagnosed deep vein thrombosis in the general population: systematic review. Eur J Vasc Endovasc Surg. 2003; 25(1): 1-5.

19. Guijarro R, Montes J, Sanroman C. Epidemiología hospitalaria de la enfermedad tromboembólica venosa en España. Med Clin (Barc) 2008; 131 Supl 2: 2-9

20. Anderson FA Jr, Spencer FA. Risk factors for venous thromboembolism. Circulation. 2003; 107(23 Suppl 1): 19-16

21. Goldhaber SZ, Tapson VF. DVT Free Steering Comittee. A prospective registry of 5451 patients with ultrasound -confirmed deep vein thrombosis. Am J Cardiol 2004; 93: 259-62

22. Goldberg RJ, Seneff M, Gore JM, Anderson FA, Greene HL, Wheeler HB, Dalen JE, Occult malignant neoplasm in patients with deep venous thrombosis. Arc Intern Med 1987; 147: 251-3

23. Monreal M, Lensing AW, Prins MH, Bonet M, Fernandez-Llamazares J, Muchart J. Screening for ocult cancer in patients with acute deep vein thrombosis or pulmonary thromboembolism. J Thromb Haemost 2004; 2: 876-81.

24. Piccioli A, Lensing AW, Prins MH, Falanga A, Scannapieco GL, leran M, Cigolini M, Ambrosio GB, Monreal M, Girolami A, Prandoni P; SOMIT Investigators Group. Extensive screening for occult malignant disease in idiopathic venous thromboembolism: a prospective randomized clinical trial. J Thromb Haemost. 2004; 2:884-9.

25. DI Nisio M, Otten HM, Piccioli A, Lensing AW, Prandoni P, Büller HR, Prins MH Decision analysis for cancer screening in idiopathic venous thromboembolism. J Thromb Haemost. 2005; 3: 2391-6.

26. Steg PG, Bhatt DL, Wilson PW, D’Agostino R, Ohmman R, Röther J. One-year cardiovascular event rates in outpatients with atherothormbosis. JAMA 2007; 297: 1197-206.

27. Lozano Sánchez FS. Epidemiología y factores de riesgo de la enfermedad arteria periférica de las extremidades periféricas en España. Med Clin Cursos (Barc) 2012 4(1): 3-8

28. Guerrero A, Montes R, Muñoz-Terol J, Gil-Peralta A, Toro J, Naranjo M, González-Pé rez P, Martín-Herrera C, Ruiz-Fernández A. Peripheral arterial disease in patients with stages IV and V chronic renal failure. Nephrol Dial Transplant. 2006;21(12):3525-31

29. Elfant AB, Levine SM, Peikin SR, Cencora B, Méndez L, Pello MJ, Atabek UM, Alexander JB, Spence RK, Camishion RC. Bioavailability of medication delivered via nasogastric tube is decreased in the immediate postoperative period. Am J Surg. 1995;169(4):430-2

30. Hamilton H, Gallagher P, Ryan C, Byrne S, O'Mahony D. Potentially inappropriate medications defined by STOPP criteria and the risk of adverse drug events in older hospitalized patients. Arch Intern Med. 2011;171(11):1013-9.

31. Holbrook A, Schulman S, Witt DM, Vandvik PO, Fish J, Kovacs MJ, Svensson PJ, Veenstra DL, Crowther M, Guyatt GH; American College of Chest Physicians. Evidence-based management of anticoagulant therapy: Antithrombotic Therapy and Prevention of Thrombosis, 9th ed: American College of Chest Physicians EvidenceBased Clinical Practice Guidelines. Chest. 2012:141(2 Suppl):52S-84S.

32. Rabuñal Rey R, Monte Secades R. Valoración de neoplasia oculta en pacientes con enfermedad tromboembólica venosa. En: R Barba Martín. Protocolos enfermedad tromboembólica venosa. Sociedad Española de Medicina Interna. Actualización 2009; capítulo 10: 141-150. Barcelona: Elsevier (ISBN 978-84-692-5840-8)

33. DeCarolis DD, Thorson JG, Clairmont MA, Leuthner AM, Rector TS, Johnson GJ. Enoxaparin outcomes in patients with moderate renal impairment. Arch Intern Med. 2012; 172(22):1713-8.

34. Nutescu EA, Spinler SA, Wittkowsky A, Dager WE. Low-molecular-weight heparins in renal impairment and obesity: available evidence and clinical practice recommendations across medical and surgical settings. Ann Pharmacother. 2009; 43(6):106483

35. Scally G, Donaldson LJ The NHS's 50 anniversary. Clinical governance and the drive for quality improvement in the new NHS in England. BMJ. 1998; 4;317(7150):61-5.

36. Jiménez-Puente A, García-Alegría J, Lara-Blanquer A. Sistemas de información para clínicos II. Cómo analizar la eficiencia y la calidad de la asistencia intrahospitalaria. Rev Clin Esp. 2010; 210(7):350-4 